\title{
Immune function genes CD99L2, JARID2 and TPO show association with autism spectrum disorder
}

\author{
Paula S Ramos ${ }^{1}$, Satria Sajuthi ${ }^{2}$, Carl D Langefeld ${ }^{2}$ and Stephen J Walker ${ }^{3^{*}}$
}

\begin{abstract}
Background: A growing number of clinical and basic research studies have implicated immunological abnormalities as being associated with and potentially responsible for the cognitive and behavioral deficits seen in autism spectrum disorder (ASD) children. Here we test the hypothesis that immune-related gene loci are associated with ASD.

Findings: We identified 2,012 genes of known immune-function via Ingenuity Pathway Analysis. Family-based tests of association were computed on the 22,904 single nucleotide polymorphisms (SNPs) from the 2,012 immunerelated genes on 1,510 trios available at the Autism Genetic Resource Exchange (AGRE) repository. Several SNPs in immune-related genes remained statistically significantly associated with ASD after adjusting for multiple comparisons. Specifically, we observed significant associations in the CD99 molecule-like 2 region (CD99L2, rs 1 1796490, $\left.P=4.01 \times 10^{-06}, \mathrm{OR}=0.68(0.58-0.80)\right)$, in the jumonji AT rich interactive domain 2 (JARID2) gene ( $r$ 13193457, $\left.P=2.71 \times 10^{-06}, \mathrm{OR}=0.61(0.49-0.75)\right)$, and in the thyroid peroxidase gene $(T P O)$ ( $r s 1514687$, $\left.P=5.72 \times 10^{-06}, \mathrm{OR}=1.46(1.24-1.72)\right)$.
\end{abstract}

Conclusions: This study suggests that despite the lack of a general enrichment of SNPs in immune function genes in ASD children, several novel genes with known immune functions are associated with ASD.

Keywords: Autism Spectrum Disorders, immune loci, family-based association analysis, single-nucleotide polymorphism

\section{Findings}

\section{Background}

Autism spectrum disorders (ASDs) encompass a heterogeneous group of clinical descriptors whose core features include deficits in cognition, communication and social acuity, coupled with stereotypical behaviors. Currently, 1 in 88 children in the United States have an ASD diagnosis, with boys being affected at a ratio of 5:1 compared to girls [1]. The etiology of ASDs, with very few exceptions, is unknown although a growing number of clinical and basic research studies have implicated immunological abnormalities as being associated with and potentially responsible for the cognitive and behavioral deficits seen in ASD children [2-4].

A judicious and efficient way to identify genetic variation predisposing to complex diseases is the application

\footnotetext{
* Correspondence: swalker@wakehealth.edu

${ }^{3}$ Wake Forest Institute for Regenerative Medicine, Wake Forest School of Medicine, 391 Technology Way, Winston-Salem, NC 27101, USA

Full list of author information is available at the end of the article
}

of a hypothesis-driven framework that incorporates prior biological knowledge. Unlike agnostic genome wide association studies (GWAS), this approach narrows the hypothesis space to provide a more focused and powerful examination of the data. Indeed, several studies have shown that a hypothesis-driven approach of selecting candidate genes serves to increase the reliability and likelihood of finding genes that are truly associated with disease [5,6]. In accord with these considerations, we tested for associations between immune-related genes and ASD children.

\section{Approach}

We used Ingenuity Pathway Analysis (IPA) [7], a bioinformatics database, to search for molecules with known immune function. IPA offers the largest and most comprehensive set of functional annotations, integrating manually curated data from other databases, and a broad range of scientific literature. Briefly, we searched for "immune" under Functions and Diseases, exported the 
molecule annotations, and used MatchMiner [8] to get the positions of the corresponding 2,012 immune-function genes (Additional file 1: Table S1).

We then used the genotypic data available at the Autism Genetic Resource Exchange (AGRE) [9] repository to perform a family-based association analysis between variants in those 2,012 immune loci and ASD. These data have previously been used in several GWAS [10-12], and the families used in our analysis are, with the exception of minor differences due to slightly different quality control criteria, the same ones as reported in Ma et al. [10], Wang et al. [11] and Weiss et al. [12]. These data and families also overlap with those reported by Voineagu et al. [13], who, included in their expression profiling study, used the AGRE data to perform an analysis searching for evidence of a genetic enrichment of immune loci. We searched for SNPs in a region including $5 \mathrm{~kb}$ up- and downstream of each immune gene. After applying quality control filters, we identified 22,904 SNPs genotyped in the AGRE collection of 1,510 trios on the Illumina Hap550 platform. We tested these SNPs for association with ASD using the standard Transmission Disequilibrium Test (TDT), as implemented in PLINK v1.07 [14]. In addition, given that the 1,510 trios are part of 1,057 independent nuclear families, we also used the Pedigree Disequilibrium Test (PDT), which uses data from related nuclear families and discordant sibships from extended pedigrees [15]. The following exclusion filters were applied: minor allele frequency $(\mathrm{MAF})<0.01$, SNP genotyping missing rate $>10 \%$, individuals $>10 \%$ missing genotypes, HardyWeinberg Equilibrium (HWE) $P$-value $<0.001$ (founders), Mendelian error rate $>5 \%$ per family and $>4 \%$ per SNP.

Since we hypothesized that ASD children may be enriched for risk loci in immune genes, we tested for a potential enrichment of significant $P$-values for SNPs in immune-related genes. We tested for an enrichment of association signals in these genes using INRICH [16], which corrects the empirical gene-set $P$-value using Bootstrapping-based re-sampling. In addition, we assessed the distribution of the linkage-disequilibrium (LD)-pruned immune-gene-set with random LD-pruned
SNPs, using an $\mathrm{r}^{2}>0.2$ for LD-based SNP pruning. We did not observe an enrichment of immune-gene associations with either test $(P=1.0$ and $P=0.45$, respectively).

In order to adjust for multiple testing we applied a Benjamini and Hochberg false discovery rate (FDR) correction [17] to the 22,904 SNPs analyzed (P-FDR). Only three SNPs remained statistically significant (P-FDR $<0.05$ ) (Table 1). Under an additive model, and at alpha $=1.0 \mathrm{E}-05$, this sample has $>80 \%$ power to detect the associations reported in Table 1.

Finally, in order to fine map the novel loci, we used BEAGLE v3.3.2 [18] and the 1000 Genomes Project Phase 1 (version 3; http://www.1000genomes.org/) reference panel to impute all SNPs in the regions of the top genes.

\section{Novel immune genes}

The lack of an enrichment of significant associations in immune genes corroborates the findings by Voineagu et al. [13], who used a complimentary approach to test for evidence of a genetic component for the up-regulation of immune response genes in the autistic brain using the same AGRE genotype data. Nevertheless, we uncovered a few associations in immune-related genes that met an FDR-adjustment.

We observed a significant association in the CD99 molecule-like 2 gene (CD99L2) (rs11796490, $P=4.01 \times 10^{-06}$, $\mathrm{OR}=0.68(0.58-0.80)$ ) (Table 1). An imputed variant in LD with this genotyped SNP showed even stronger association $\left(\right.$ rs7880807, $\left.P=8.26 \times 10^{-07}, \mathrm{OR}=0.64(0.53-0.76)\right)\left(\mathrm{r}^{2}=1.0\right.$ in CEU from HapMap release 21) (Figure 1). As is evident from Figure 1, other variants in this gene are also showing evidence of association with ASD. Although more modest, these associations with neighboring SNPs corroborate the association observed at rs11796490, which is, therefore, less likely to be spurious. This variant is located in the first intron of CD99L2. The product of this gene functions as an adhesion molecule during leukocyte extravasation, in particular at the diapedesis step.

A variant in the first intron of the jumonji AT rich interactive domain 2 (JARID2) gene showed association

Table 1 Most significant immune loci from the association analyses

\begin{tabular}{|c|c|c|c|c|c|c|c|c|c|}
\hline \multirow[t]{2}{*}{ SNP } & \multirow[t]{2}{*}{ Chr } & \multirow{2}{*}{$\begin{array}{l}\text { Pos } \\
\text { (Mb) }\end{array}$} & \multirow[t]{2}{*}{ Region } & \multicolumn{5}{|l|}{ TDT } & \multirow{2}{*}{$\begin{array}{l}\text { PDT } \\
\text { P-value }\end{array}$} \\
\hline & & & & $\overline{\mathrm{MA}}$ & $\mathrm{T}: \mathrm{U}$ & OR $(95 \% \mathrm{Cl})$ & $P$-value & P-FDR & \\
\hline rs7880807' & $x$ & 15.006 & CD99L2 & A & $190: 299$ & $0.64(0.53-0.76)$ & $8.26 \mathrm{E}-07$ & - & ND \\
\hline rs13193457* & 6 & 15.346 & JARID2 & A & $139: 229$ & $0.61(0.49-0.75)$ & $2.71 \mathrm{E}-06$ & - & $6.04 \mathrm{E}-05$ \\
\hline rs11796490 & $X$ & 150.058 & CD99L2 & G & 239:351 & $0.68(0.58-0.80)$ & 4.01E-06 & $1.53 \mathrm{E}-02$ & NA \\
\hline rs1514687 & 2 & 1.438 & $\mathrm{TPO}$ & A & $349: 239$ & $1.46(1.24-1.72)$ & $5.72 \mathrm{E}-06$ & 1.87E-02 & 4.49E-06 \\
\hline rs10890518' & 2 & 1.435 & TPO & G & $356: 249$ & $1.43(1.22-1.62)$ & $1.36 \mathrm{E}-05$ & - & ND \\
\hline
\end{tabular}

Chr, Chromosome; Pos., position; MA, minor allele; T:U, transmitted to untransmitted minor allele counts; OR, odds ratio; Cl, confidence interval; P-FDR. False Discovery Rate-adjusted $P$-value; ND, test not done with imputed data; NA, not applicable, as the PDT does not handle chromosome X data. ${ }^{*}$ This SNP had a 5\% Mendelian error rate. 'This SNP was imputed. 


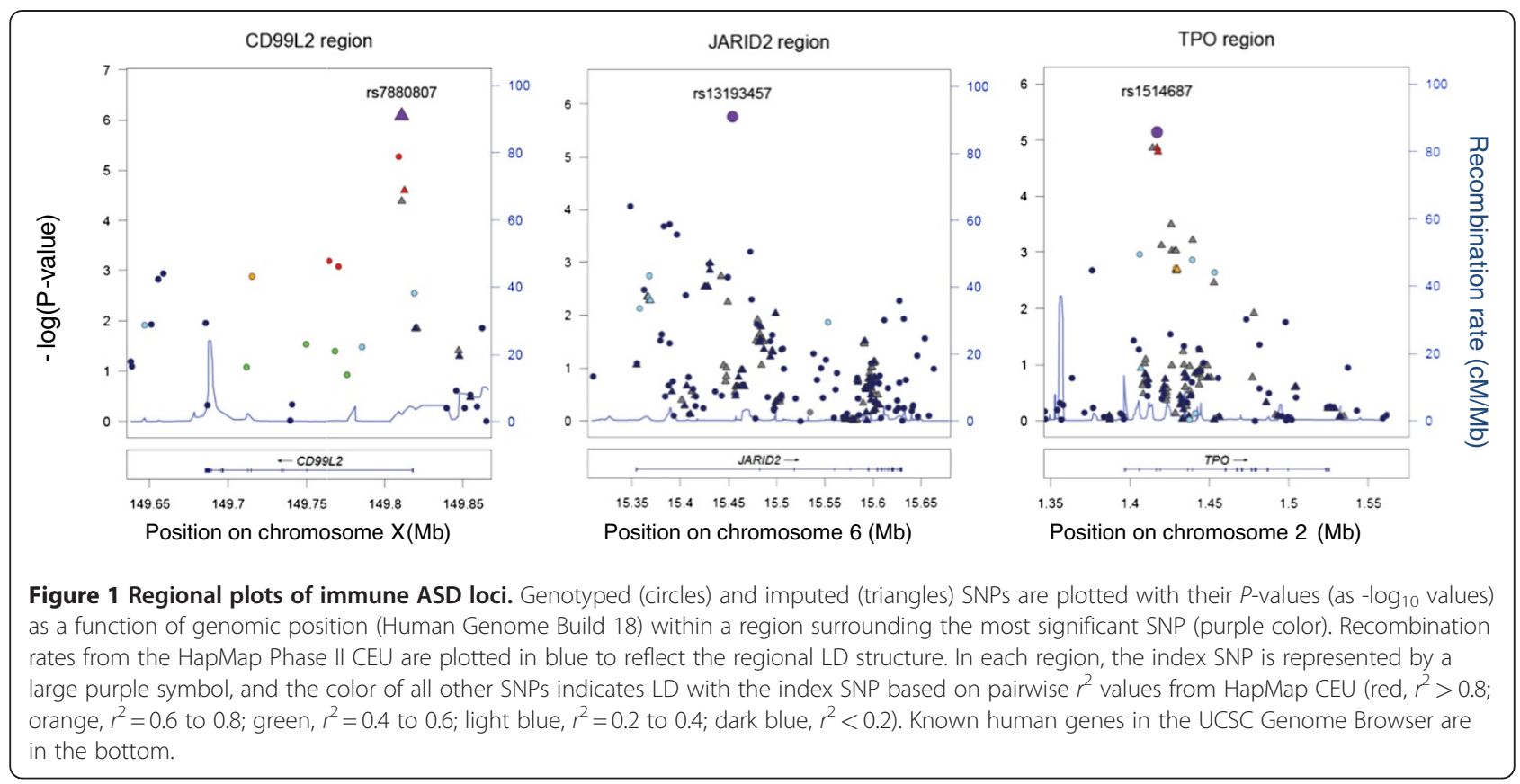

with ASD (rs13193457, $P=2.71 \times 10^{-06}, \mathrm{OR}=0.61(0.49$ 0.75) (Table 1). Despite having a borderline Mendelian error rate, this SNP is of interest. This gene has been previously implicated in schizophrenia $[19,20]$ and, more recently, it has been reported in a GWAS for ASD that also included the same AGRE dataset herein used [12]. The variant reported by Weiss et al. (rs7766973) does not show LD with rs13193457 $\left(\mathrm{r}^{2}=0.13\right.$ in CEU from 1000 Genomes Pilot 1). The jumonji-domain functions by removing methyl marks on histones that are associated with gene regulation [21]. JARID2 is required for neural tube formation and is essential for normal heart development and function, as well as acting as a transcriptional repressor of ANF by binding to both GATA4 and NKX2-5 and repressing their transcriptional activator activities.

An intronic variant in the thyroid peroxidase gene (TPO) has also shown association with ASD (rs1514687, $\left.P=5.72 \times 10^{-06}, \mathrm{OR}=1.46(1.24-1.72)\right)$. This association is corroborated by those of neighboring SNPs (Figure 1). This gene has been associated with hypothyroidism [22]. This gene encodes a membrane-bound glycoprotein which acts as an enzyme and plays a central role in thyroid gland function. The protein functions in the iodination of tyrosine residues in thyroglobulin and phenoxyester formation between pairs of iodinated tyrosines to generate the thyroid hormones, thyroxine and triiodothyronine. Mutations in this gene are associated with several disorders of thyroid hormonogenesis, including congenital hypothyroidism, congenital goiter and thyroid hormone organification defect IIA. Multiple transcript variants encoding distinct isoforms have been identified for this gene.

\section{Discussion}

In spite of its reported high heritability, the identification of genetic risk factors predisposing to ASDs has been difficult. To date, only a small number of loci are considered established (that is, have met genome-wide significance and have been replicated in independent cohorts). GWAS are by design agnostic. Increased statistical power can be achieved with more focused hypotheses. Given the increasing speculation of a significant role for immune genes in the etiology and pathogenesis of ASD, we undertook a search for genetic variation associated with ASD in genes with immune functions. This hypothesis-driven candidate gene approach based on prior knowledge was selected because it serves to increase the power, reliability and likelihood of finding genes that are associated with disease $[5,6]$.

A lack of enrichment of genetic associations in immune-related genes has recently been reported [13] in the same dataset we used, and this conclusion is supported here using another statistical approach. The associations we uncovered in immune-related genes and the observed dysregulation of immune genes in ASD [13] suggest that variation in a limited number of immunefunction genes may be responsible for observed up-regulation of their immune downstream targets.

The transmission/disequilibrium test (TDT) is largely robust to population substructure as it is a within-family comparison. The individuals in these data are predominately of European descent (76\%), with Asian (3\%) and African (2\%) ancestry represented; the Autism Genetic Resource Exchange (AGRE) repository lists $14 \%$ and $5 \%$ of the individuals 
of unknown or admixed ancestry. One limitation of using the TDT in our study is the lack of total independence between the trios analyzed: the 1,510 trios used in the TDT are part of 1,101 independent nuclear families. The correlation among children in the same family can inflate the type I error probability. We, thus, also applied the PDT, also a within family test that is robust to population substructure, and the PDT analysis corroborates the TDT analysis.

\section{Conclusion}

SNPs within the CD99 molecule-like 2 gene, the jumonji AT rich interactive domain 2 (JARID2) gene, and the thyroid peroxidase gene were associated with ASD after multiple comparisons adjustment. Understanding how these genetic factors might contribute to pathogenesis should ultimately lead to important opportunities for discovering therapeutic targets that can be used to treat ASD.

\section{Additional file}

Additional file 1: Table S1. Immune-function genes.

\section{Abbreviations \\ AGRE: Autism Genetic Resource Exchange; ASDs: Autism Spectrum Disorders; $\mathrm{Cl}$ : confidence interval; FDR: False Discovery Rate; GWAS: genome wide association studies; HWE: Hardy-Weinberg Equilibrium; IPA: Ingenuity Pathway Analysis; JARID2: jumonji AT rich interactive domain 2; MAF: minor allele frequency; OR: odds ratio; PDT: Pedigree Disequilibrium Test; P-FDR: False Discovery Rate-adjusted P-value; SNP: single nucleotide polymorphism; TDT: Transmission Disequilibrium Test; TPO: thyroid peroxidase gene.}

\section{Competing interests}

The authors declare that they have no competing interests.

\section{Acknowledgements}

We gratefully acknowledge the resources provided by the Autism Genetic Resource Exchange (AGRE) Consortium* and the participating AGRE families. The Autism Genetic Resource Exchange is a program of Autism Speaks and is supported, in part, by grant 1U24MH081810 from the National Institute of Mental Health to Clara M. Lajonchere (PI). We also acknowledge funding from the Jane Botsford Johnson Foundation, The Thoughtful House Center for Children, and the Wake Forest School of Medicine Center for Public Health Genomics.

\section{Author details}

'Division of Rheumatology and Immunology, Department of Medicine, Medical University of South Carolina, 96 Jonathan Lucas St., Suite 912, Charleston, SC 29425, USA. ${ }^{2}$ Department of Biostatistical Sciences and Center for Public Health Genomics, Division of Public Health Sciences, Wake Forest School of Medicine, Medical Center Boulevard, WC 23, Winston Salem, NC 27157, USA. ${ }^{3}$ Wake Forest Institute for Regenerative Medicine, Wake Forest School of Medicine, 391 Technology Way, Winston-Salem, NC 27101, USA.

\section{Authors' contributions}

PSR conceived the study, participated in the design of the study, performed statistical analysis and results interpretation, and drafted the manuscript. SS performed statistical analysis. CDL conceived the study, participated in the design of the study, coordinated the study and drafted the manuscript. SJW conceived the study, participated in its design and drafted the manuscript. All authors read and approved the final manuscript.
Received: 16 December 2011 Accepted: 9 June 2012

Published: 9 June 2012

\section{References}

1. CDC: Prevalence of autism spectrum disorders - Autism and Developmental Disabilities Monitoring Network, United States. Morb Mortal Wkly Rep Surveill Summ 2012, 61:1-19.

2. Ashwood P, Krakowiak P, Hertz-Picciotto I, Hansen R, Pessah I, Van de Water $\mathrm{J}$ : Elevated plasma cytokines in autism spectrum disorders provide evidence of immune dysfunction and are associated with impaired behavioral outcome. Brain Behav Immun 2011, 25:40-45.

3. Jyonouchi H, Geng L, Streck DL, Toruner GA: Children with autism spectrum disorders (ASD) who exhibit chronic gastrointestinal (Gl) symptoms and marked fluctuation of behavioral symptoms exhibit distinct innate immune abnormalities and transcriptional profiles of peripheral blood (PB) monocytes. J Neuroimmunol 2011, 238:73-80.

4. Onore $C$, Careaga $M$, Ashwood P: The role of immune dysfunction in the pathophysiology of autism. Brain Behav Immun 2012, 26:383-392.

5. Armstrong DL, Reiff A, Myones BL, Quismorio FP Jr, Klein-Gitelman M, McCurdy D, Wagner-Weiner L, Silverman E, Ojwang JO, Kaufman KM, Kelly JA, Merrill JT, Harley JB, Bae SC, Vyse TJ, Gilkeson GS, Gaffney PM, Moser KL, Putterman C, Edberg JC, Brown EE, Ziegler J, Langefeld CD, Zidovetzki R, Jacob CO: Identification of new SLE-associated genes with a two-step Bayesian study design. Genes Immun 2009, 10:446-456.

6. Gaulton KJ, Willer CJ, Li Y, Scott LJ, Conneely KN, Jackson AU, Duren WL, Chines PS, Narisu N, Bonnycastle LL, Luo J, Tong M, Sprau AG, Pugh EW, Doheny KF, Valle TT, Abecasis GR, Tuomilehto J, Bergman RN, Collins FS, Boehnke M, Mohlke KL: Comprehensive association study of type 2 diabetes and related quantitative traits with 222 candidate genes. Diabetes 2008, 57:3136-3144.

7. Ingenuity Pathway Analysis. http://www.ingenuity.com.

8. MatchMiner., http://discover.nci.nih.gov/matchminer/index.jsp.

9. Autism Genetic Resource Exchange. http://research.agre.org.

10. Ma D, Salyakina D, Jaworski JM, Konidari I, Whitehead PL, Andersen AN, Hoffman JD, Slifer SH, Hedges DJ, Cukier HN, Griswold AJ, McCauley JL, Beecham GW, Wright HH, Abramson RK, Martin ER, Hussman JP, Gilbert JR, Cuccaro ML, Haines JL, Pericak-Vance MA: A genome-wide association study of autism reveals a common novel risk locus at $5 \mathrm{p} 14.1$. Ann Hum Genet 2009, 73:263-273.

11. Wang K, Zhang H, Ma D, Bucan M, Glessner JT, Abrahams BS, Salyakina D, Imielinski M, Bradfield JP, Sleiman PM, Kim CE, Hou C, Frackelton E, Chiavacci R, Takahashi N, Sakurai T, Rappaport E, Lajonchere CM, Munson J, Estes A, Korvatska O, Piven J, Sonnenblick LI, Alvarez Retuerto Al, Herman El, Dong H, Hutman T, Sigman M, Ozonoff S, Klin A, et al: Common genetic variants on 5 p14.1 associate with autism spectrum disorders. Nature 2009, 459:528-533.

12. Weiss $L A$, Arking DE, Daly MJ, Chakravarti A: A genome-wide linkage and association scan reveals novel loci for autism. Nature 2009, 461:802-808.

13. Voineagu I, Wang X, Johnston P, Lowe JK, Tian Y, Horvath S, Mill J, Cantor RM, Blencowe BJ, Geschwind DH: Transcriptomic analysis of autistic brain reveals convergent molecular pathology. Nature 2011, 474:380-384.

14. Purcell S, Neale B, Todd-Brown K, Thomas L, Ferreira MA, Bender D, Maller J, Sklar P, de Bakker PI, Daly MJ, Sham PC: PLINK: a tool set for whole-genome association and population-based linkage analyses. Am J Hum Genet 2007, 81:559-575.

15. Martin ER, Monks SA, Warren LL, Kaplan NL: A test for linkage and association in general pedigrees: the pedigree disequilibrium test. Am J Hum Genet 2000, 67:146-154.

16. INRICH. http://atgu.mgh.harvard.edu/inrich/.

17. Benjamini $Y$, Hochberg $Y$ : Controlling the false discovery rate: a practical and powerful approach to multiple testing. J R Stat SoC B 1995, 57:289-300

18. Browning BL, Browning SR: A unified approach to genotype imputation and haplotype-phase inference for large data sets of trios and unrelated individuals. Am J Hum Genet 2009, 84:210-223.

19. Pedrosa E, Ye K, Nolan KA, Morrell L, Okun JM, Persky AD, Saito T, Lachman HM: Positive association of schizophrenia to JARID2 gene. Am J Med Genet B Neuropsychiatr Genet 2007, 144B:45-51.

20. Liu Y, Chen G, Norton N, Liu W, Zhu H, Zhou P, Luan M, Yang S, Chen X, Carroll L, Williams NM, O'Donovan MC, Kirov G, Owen MJ: Whole genome association study in a homogenous population in Shandong peninsula 
of China reveals JARID2 as a susceptibility gene for schizophrenia. J Biomed Biotechnol 2009, 2009:536918.

21. Cloos PA, Christensen J, Agger K, Helin K: Erasing the methyl mark: histone demethylases at the center of cellular differentiation and disease. Genes Dev 2008, 22:1115-1140.

22. Avbelj M, Tahirovic H, Debeljak M, Kusekova M, Toromanovic A, Krzisnik C, Battelino T: High prevalence of thyroid peroxidase gene mutations in patients with thyroid dyshormonogenesis. Eur J Endocrinol 2007, 156:511-519.

doi:10.1186/2040-2392-3-4

Cite this article as: Ramos et al: Immune function genes CD99L2, JARID2

and TPO show association with autism spectrum disorder. Molecular

Autism 2012 3:4.

\section{Submit your next manuscript to BioMed Central and take full advantage of:}

- Convenient online submission

- Thorough peer review

- No space constraints or color figure charges

- Immediate publication on acceptance

- Inclusion in PubMed, CAS, Scopus and Google Scholar

- Research which is freely available for redistribution 